\title{
Rupture of distal anterior cerebral artery aneurysm presenting only subdural hemorrhage without subarachnoid hemorrhage: a case report
}

Tae-Wook Song, Sung-Hyun Kim, Seung-Hoon Jung, Tae-Sun Kim and Sung-Pil Joo*

\begin{abstract}
Intracranial aneurysm rupture usually manifests with subarachnoid hemorrhage, often combined with intracerebral hemorrhage with intraventricular hemorrhage extension. In rare cases, however, these aneurysms present only as subdural hematomas. Recently, we treated a 48-years-old female patient who presented only with subdural hematoma. Interestingly, she did not have a history of trauma. Computed tomography angiography and digital subtraction angiography revealed a $5 \times 3 \mathrm{~mm}$ sized aneurysm at the A3-A4 junction of the left anterior cerebral artery. On admission, emergency operation (clipping and hematoma evacuation) was performed to protect against re-bleeding. Along with postoperative intensive care, the patient returned to normal daily life with only a mild headache. Given that patients may present with atraumatic acute subdural hematoma, the clinician must bear in mind the possibility of intracranial vascular pathology and obtain angiographic scans to evaluate for any underlying conditions to prevent patient deaths.
\end{abstract}

Keywords: Spontaneous subdural hematoma, Ruptured aneurysm, Subarachnoid hemorrhage

\section{Introduction}

Aneurysm rupture usually presents as a subarachnoid hemorrhage (SAH), often in combination with intracerebral hemorrhage (ICH) and extension into the ventricle (intraventricular hemorrhage, IVH) (Barton and Tudor 1982; Mansour et al. 2014). In rare cases, acute subdural hematoma (aSDH) combined with SAH due to aneurysm rupture has been reported (Biesbroek et al. 2012, 2013; Kocak et al. 2009; Marbacher et al. 2010; Nozar et al. 2002), with only a few cases reported since the first case was reported in 1855 (Kocak et al. 2009; Marbacher et al. 2010). According to the literature, the incidence of aSDH due to aneurysmal rupture varies from 0.5 to $22 \%$ (Barton and Tudor 1982; Biesbroek et al. 2012; Marbacher et al. 2010). The majority of patients with aSDH have

\footnotetext{
*Correspondence: nsjsp@chonnam.ac.kr; nsjsp@hanmail.net Department of Neurosurgery, Chonnam National University Hospital and Medical School, 42, Jebong-ro, Donggu, Gwangju 501-757, Republic of Korea
}

sustained trauma, directly responsible for injury to bridging or cortical veins that ultimately leads to the subdural collection of blood with SAH combined with aSDH a have worse prognosis than patients without aSDH (Biesbroek et al. 2013). Uncommonly, other causes of spontaneous aSDH include arteriovenous malformations, cocaine abuse, neoplasms, and dural arteriovenous fistulars, to name a few (Alves and Gomes 2000).

Here we report an interesting case of spontaneous aSDH without a history of trauma due to aneurysmal rupture at the A3-A4 junction of the anterior cerebral artery (ACA). In addition, we elaborate on possible mechanisms of spontaneous aSDH without head trauma.

\section{Case report}

A 48-years-old female presented to a local hospital complaining of a severe headache and underwent a brain computed-tomography (CT) scan which revealed an acute subdural hematoma. She was transferred to our hospital for further evaluation and treatment. Despite a 
severe headache, the patient had an alert mental status; more interestingly, the patient also denied a history of trauma. Beside hypertension, which was well-controlled with a combination of a calcium channel blocker and an angiotensin receptor blocker, the patient had no medical or surgical history, and took no other medication, including anticoagulants. Preoperative coagulation tests confirmed the lack of anticoagulant use as they were normal. Brain computed tomography (CTA) and digital subtraction angiography (DSA) were performed to evaluate for vascular abnormalities, and these angiographic studies revealed a $5 \times 3 \mathrm{~mm}$ sized aneurysm at the A3-A4 junction of left ACA (Fig. 1). On admission to the hospital, to protect against re-bleeding, the aneurysm was clipped through a unilateral interhemispheric approach via a bicoronal craniotomy and the SDH was evacuated. Cerebrospinal fluid was noted to be clear and free of blood products. SAH was not observed at the aneurysm site, with only the rupture point detectable at the site of the aneurysm (Fig. 2). Following the emergency operation, the patient's treatment continued in the intensive care unit, from which she was transferred to the floor on postoperative day seven. The patient walked unaided out of the hospital 2 weeks after surgery, returning to her normal, daily routine and only complaining of

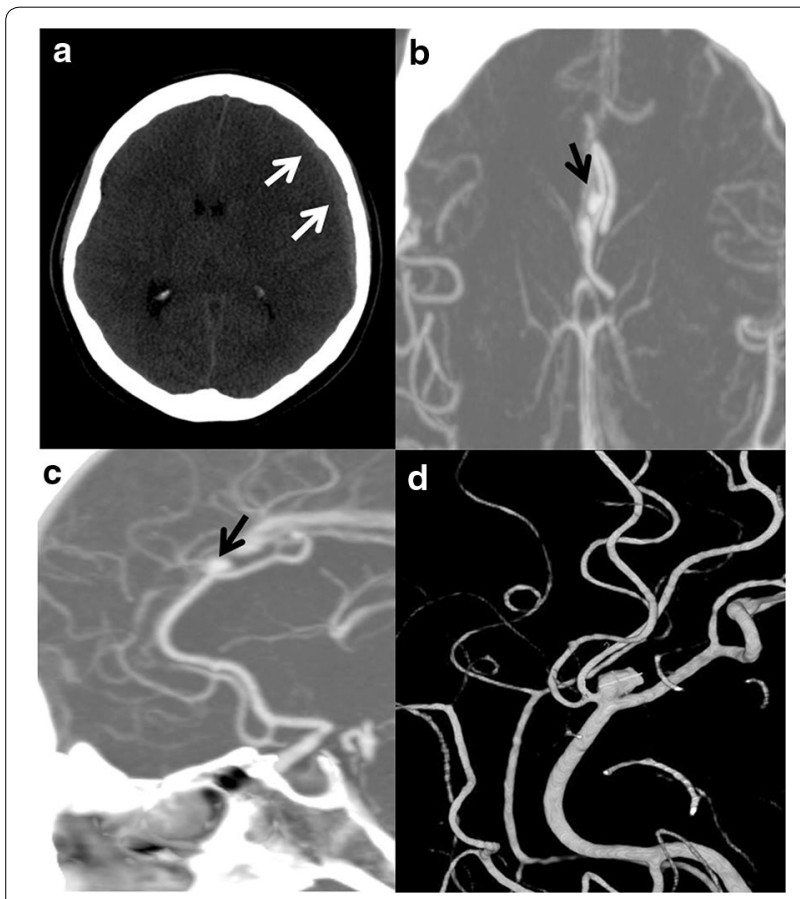

Fig. 1 a Non-contrast brain CT shows acute subdural hematoma in the left fronto-tempo-parietal area (white arrow). The midline is shifted about $6 \mathrm{~mm}$ to the right. b, c Brain CTA reveals an A3-A4 junction aneurysm (black arrow). d 3D-DSA reveals an aneurysm at the A3-A4 junction a mild headache. The patient did not have any complications after surgery. Follow up brain CTA revealed that the previous subdural hematoma was evacuated and the aneurysm at the A3-A4 junction was clipped without a remnant sac (Fig. 3). Moreover, the patient's Glasgow Outcome Scale (GOS) measured five points 9 months after surgery.

\section{Discussion}

The majority of aSDH cases (95\%) are associated with head injuries (Marbacher et al. 2010; O'Leary and Sweeny 1986). A brain CT scan allows bleeding to be distinguished from other causes such as hemorrhagic contusions, skull fractures, and punctuate hemorrhage (Marbacher et al. 2010). Detection of a hemorrhage in a common aneurysm site that presents as SAH can allow for discovering the origin of the bleed (Ohkuma et al. 2003). In the annals of neurosurgery, only a few cases of intracranial aneurysm rupture have been reported to manifest solely as an aSDH without SAH (Nonaka et al. 2000). While we are not aware of specific radiologic features of aneurysmal aSDH reported in the literature (Marbacher et al. 2010), we nonetheless posit that for patients who present with aSDH without a history of head trauma, an aneurysmal rupture should be included in the differential diagnosis.

There are several mechanisms to explain the generation of aSDH after aneurysm rupture: (1) The rupture of the aneurysmal causes small bleeding with adhesions to the arachnoid membrane, and secondary aneurysmal rupture leads to a direct bleed into the subdural space (Kocak et al. 2009). (2) High systolic pressure during aneurysmal rupture directly breaks the arachnoid membrane and generates hematomas in the subdural space (Kocak et al. 2009). (3) Rapid accumulation of blood under pressure from the leaking aneurysm passes into the subarachnoid membrane (Kocak et al. 2009). (4) Acute enlargement of an intra-cavernous aneurysm results in erosion of the cavernous sinus wall (Nozar et al. 2002). Subsequently, rupture of this lesion causes subdural hematoma (Kocak et al. 2009). (5) A distal ACA aneurysm primarily adherent to the falx or the dura bleeds into the subdural space upon rupture (Barton and Tudor 1982; Hatayama et al. 1994; Mansour et al. 2014; Oh et al. 2011).

In the present case, we hold that the fifth mechanism described above is responsible for the subdural hematoma in our patient. Similar to other reports about ACA aneurysm rupture responsible for aSDH without SAH (Table 1) (Hatayama et al. 1994; Weil et al. 2010), during surgery, the sac of the ruptured ACA aneurysm was glued to the anterior falx with surrounding traces of hemorrhage. A relatively fresh hematoma was observed in the subdural space and was evacuated as much as possible. In 

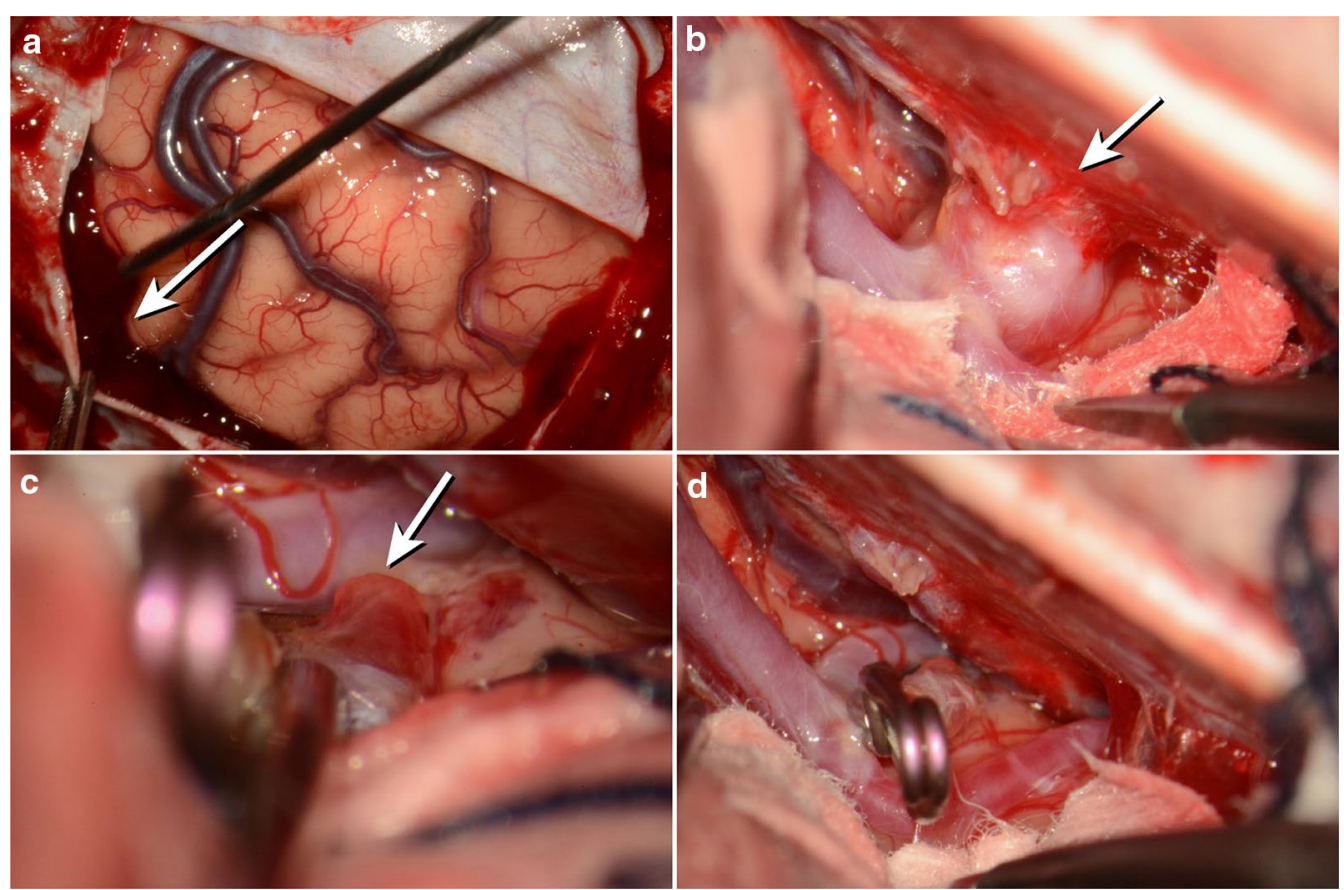

Fig. 2 a Hematoma in the subdural space (arrow). b Aneurysm adhering to the falx (arrow). c Ruptured bled (arrow). d Complete clipping state

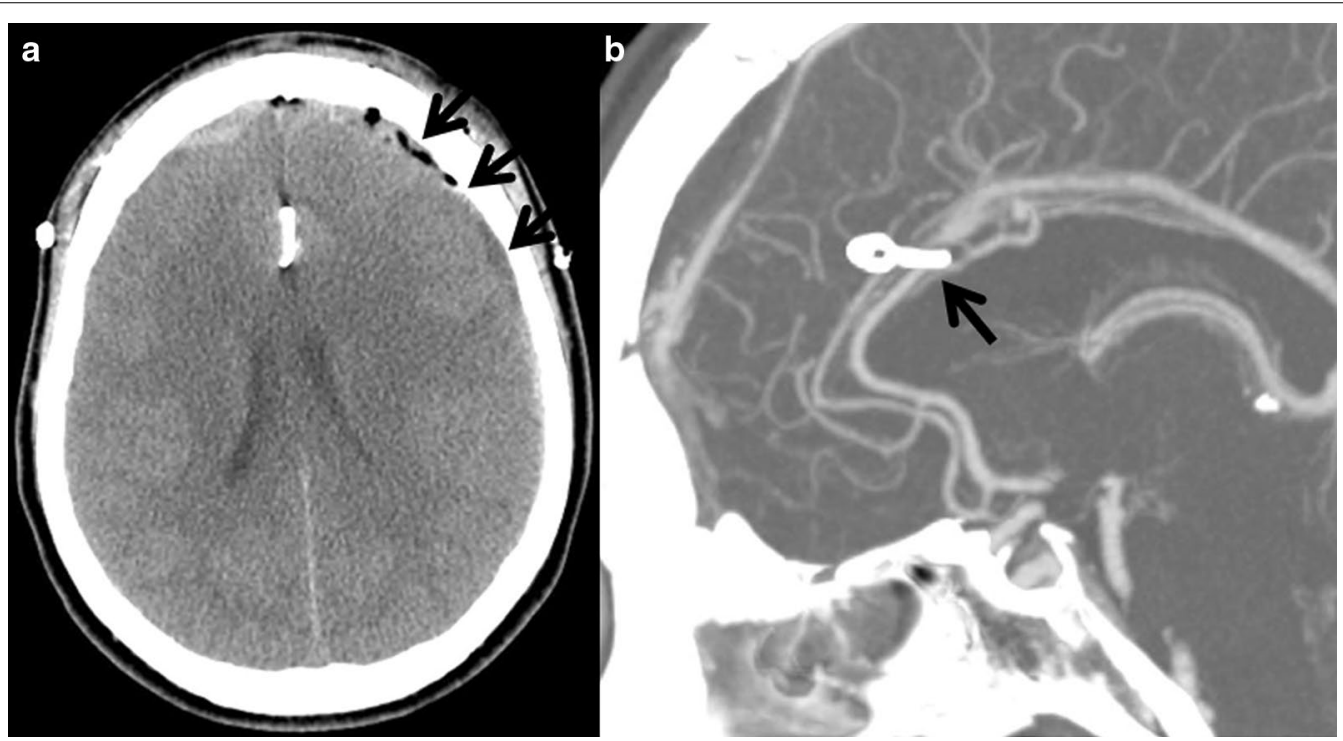

Fig. 3 a, b Postoperative CTA revealed that previous subdural hematoma was evacuated and aneurysm was clipped without a remnant sac (arrow)

rare cases, a ruptured aneurysm adhering tightly to the falx results in pure aSDH without SAH. In addition, pure aSDH without SAH is rarely induced by the rupture of a cortical aneurysm or a giant aneurysm extending into the subdural space (Hori et al. 2005; Nonaka et al. 2000; Yasui et al. 1995) (Fig. 4).
From previous reports, the incidence of aneurysmal rupture with $\mathrm{aSDH}$ is dependent on the location of the ruptured aneurysm (Cho et al. 2011; Mansour et al. 2014; Marbacher et al. 2010). In cases such as these, O'Sullivan et al. (1994) reported the location to be at the internal carotid artery (ICA) in $43 \%$, the middle cerebral artery $(\mathrm{MCA})$ in $22 \%$, the anterior communicating artery 
Table 1 Summary of the cases of pure aSDH (without SAH) caused by ACA aneurysm rupture

\begin{tabular}{|c|c|c|c|c|c|c|}
\hline Authors & Age/sex & $\begin{array}{l}\text { Signs and } \\
\text { symptoms }\end{array}$ & Site of aSDH & Site of aneurysm & Treatment & $\begin{array}{l}\text { Outcome } \\
\text { (GOS) }\end{array}$ \\
\hline Watanabe et al. (1991) & $51, M$ & Coma & $\begin{array}{l}\text { Convexity and interhemi- } \\
\text { spheric }\end{array}$ & Distal ACA & Evacuation and clipping & $\mathrm{D}(1)$ \\
\hline Ragland et al. (1993) & $55, M$ & Coma & Convexity & AcomA & Evacuation & $\mathrm{D}(1)$ \\
\hline Hatayama et al. (1994) & $55, M$ & Coma & $\begin{array}{l}\text { Convexity and interhemi- } \\
\text { spheric }\end{array}$ & Distal ACA & Evacuation and clipping & $\mathrm{GR}(5)$ \\
\hline Hatayama et al. (1994) & $66, F$ & Coma & $\begin{array}{l}\text { Convexity and interhemi- } \\
\text { spheric }\end{array}$ & Distal ACA & Evacuation and clipping & MD (4) \\
\hline Katsuno et al. (2003) & $63, F$ & Headache, nausea & $\begin{array}{l}\text { Convexity and interhemi- } \\
\text { spheric }\end{array}$ & Distal ACA & Evacuation and clipping & GR (5) \\
\hline Gilad et al. (2007) & $47, M$ & Nausea, vomiting & Sella, spinal canal & AcomA & Coiling $^{\mathrm{a}}$ & $\mathrm{GR}(5)$ \\
\hline Tomaya et al. (2012) & $54, M$ & Headache, nausea & Convexity and tentorium & A1-A2 junction & Evacuation and clipping & $\mathrm{GR}(5)$ \\
\hline Present case 2015 & $48, F$ & Headache & $\begin{array}{l}\text { Convexity and interhemi- } \\
\text { spheric }\end{array}$ & Distal ACA & Evacuation and clipping & $\mathrm{GR}(5)$ \\
\hline
\end{tabular}

AcomA anterior communicating artery, $A C A$ anterior cerebral artery, $a S D H$ acute subdural hematoma, $S A H$ subarachnoid hemorrhage, $A 1-A 2=$ anterior cerebral artery 1-2 portion, GOS Glasgow outcome scale [D = dead (1), PVS persistent vegetative state (2), SD severe disability (3), MD moderate disability (4), GR good recovery (5)]

a The patient underwent only coiling and was discharged 5 days afterward

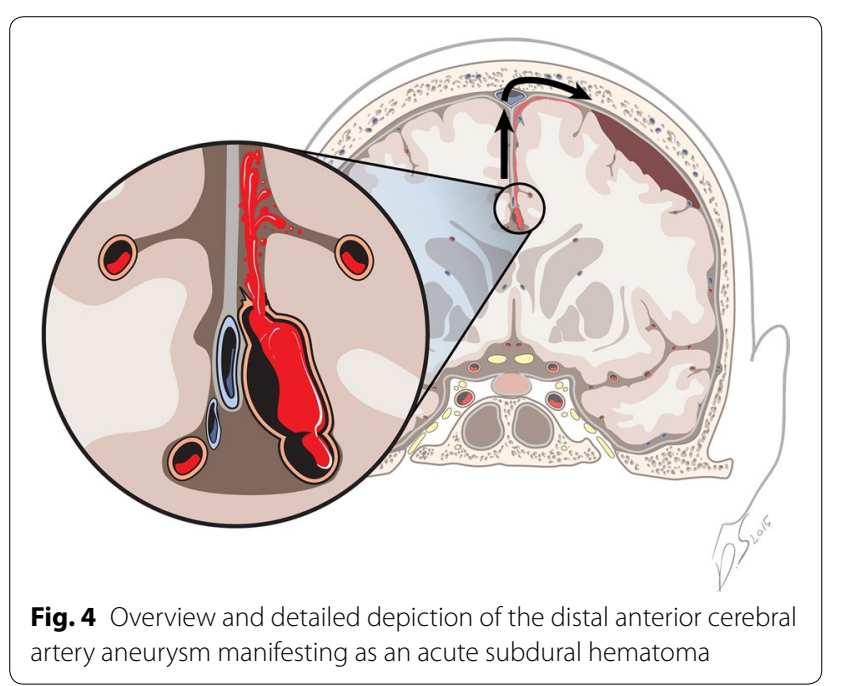

(AcomA) in $22 \%$, the vertebrobasilar artery in $4 \%$, and in other locations in $9 \%$ of cases. In slight contrast, Ohkuma et al. (2003) found the location to be at the ICA in $38 \%$, the MCA in $46 \%$, and A-com/ACA in $15 \%$ of cases, while Strang et al. reported locations at the ICA in $53 \%$, and the ACA in $27 \%$ of cases (Marbacher et al. 2010).

Because a segment of the ICA courses to the Circle of Willis through the subdural space, ICA aneurysm ruptures have a greater tendency to result in subdural hematomas (SDH) (Marbacher et al. 2010). The perianeurysmal environment associated with ICA aneurysm rupture leads to a specific bleeding pattern. It could be that an aneurysm invading the basilar cisterns makes an $\mathrm{aSDH}$, for example, in ICA or AcomA cases. However, when an aneurysm is surrounded by brain tissue, as in the cases of MCA aneurysms, rupture more likely results in SAH, ICH or IVH without an aSDH (Schuss et al. 2013). It is exceedingly rare for a posterior circulation aneurysm rupture, such as a vertebrobasilar artery aneurysm, to prevent as an aSDH due to Lilliquist's membrane surrounding the basilar artery: this membrane is thicker than the arachnoid membrane at other parts of the neurovasculature, and as such, seldom allows for bleeding into the subdural space (Biesbroek et al. 2012).

Patients with spontaneous aSDH due to the rupture of aneurysm had worse clinical outcomes than those without aSDH (Biesbroek et al. 2013; Marbacher et al. 2010). Matthjis et al. demonstrated that the rate of poor outcome in patients with aSDH due to aneurysm rupture was $79 \%$ on admission and $75 \%$ at 3 months after discharge, whereas the rate for the non-aSDH group was $50 \%$ on admission and $35 \%$ at 3 months after discharge (Biesbroek et al. 2013). Besides, the group with aSDH due to aneurysm rupture had a higher mortality than the group with only traumatic aSDH (Mansour et al. 2014), with the main reason for deterioration attributed to increased intracranial pressure (Biesbroek et al. 2013). In most cases, preoperative herniation and greater shift in the midline in the setting of large hematomas already existed (Weir et al. 1984). Accordingly, urgent decompression is required to prevent severe deterioration. As a rule, clinical outcomes correlated with the initial clinical and radiological status of each patient; good condition on admission meant a good prognosis at discharge.

The treatment strategy of spontaneous aSDH depends on the patient's initial condition (Marbacher et al. 
2010). Should the patient present with spontaneous aSDH causing life-threatening brain swelling and herniation, urgent evacuation and decompression must be performed first before further evaluations about vascular abnormalities occur (Mansour et al. 2014; Oh et al. 2011; Ohkuma et al. 2003; Schuss et al. 2013), as this emergency surgery prevents a poor outcome (Schuss et al. 2013). After recovery, further evaluation utilizing angiographic studies such as brain CTA or DSA can be considered. Subsequently, aneurysm clipping or coil embolization must be carried out in accordance with the site of the aneurysm. If the condition of patient is stable, an evaluation for vascular abnormalities should be conducted before surgical treatment (Mansour et al. 2014; Oh et al. 2011). DSA is more accurate, but brain CTA has advantages of shorter exam time and relatively good scanning quality (Kocak et al. 2009). After the aneurysm has been observed in brain images, aneurysm clipping and hematoma evaluation proceed. Following surgical treatment, coli embolization may be performed depending on the site of the aneurysm (O'Sullivan et al. 1994). If the SDH is small, coil embolization without hematoma evacuation may be performed (Marbacher et al. 2010). As with some authors, since about $90 \%$ of aneurysmal SAH is generated in the anterior circulation, explorative craniotomy should be considered for patients suffering from a spontaneous aSDH (Weir et al. 1984).

\section{Conclusion}

Spontaneous SDH due to the rupture of an intracranial aneurysm is rare. It is worth nothing, however, that the clinical outcomes of patients who present in this way are poor, thereby necessitating the need for aneurysm rupture to be considered in the differential diagnosis in patients presenting with spontaneous SDH. CTA or DSA may be helpful to detect vascular abnormalities in patients who present with acute subdural hematoma without a history of trauma.

\section{Authors' contributions}

SPJ and TWS performed the operation and evaluated the patient. SHK revised the manuscript and evaluated the patient. SHJ and TSK reviewed the chart and published literature. All authors read and approved the final manuscript.

\section{Acknowledgements}

This study was supported by a Grant (CRI 13902-21) from the Chonnam National University Hospital Biomedical Research Institute.

\section{Competing interests}

The authors declare that they have no competing interests.

Patient consent The patient and guardian have consented to the submission of this case report (IRB of Chonnam National University Hospital).

Received: 6 August 2015 Accepted: 15 January 2016

Published online: 26 January 2016

\section{References}

Alves OL, Gomes O (2000) Cocaine-related acute subdural hematoma: an emergent cause of cerebrovascular accident. Acta Neurochir 142:819-821

Barton E, Tudor J (1982) Subdural haematoma in association with intracranial aneurysm. Neuroradiology 23:157-160

Biesbroek JM, Rinkel GJ, Algra A, van der Sprenkel JW (2012) Risk factors for acute subdural hematoma from intracranial aneurysm rupture. Neurosurgery 71:264-268 (discussion 268-269)

Biesbroek JM, van der Sprenkel JW, Algra A, Rinkel GJ (2013) Prognosis of acute subdural haematoma from intracranial aneurysm rupture. J Neurol Neurosurg Psychiatry 84:254-257

Cho WS, Batchuluun B, Lee SJ, Kang HS, Kim JE (2011) Recurrent subdural hematoma from a pseudoaneurysm at the cortical branch of the middle cerebral artery after mild head injury: case report. Neurol Med Chir 51:217-221

Gilad R et al (2007) Migrating subdural hematoma without subarachnoid hemorrhage in the case of a patient with a ruptured aneurysm in the intrasellar anterior communicating artery. AJNR Am J Neuroradiol 28(10):2014-2016

Hatayama T, Shima T, Okada Y, Nishida M, Yamane K, Okita S, Yoshida A, Noae Y, Shiga N (1994) Ruptured distal anterior cerebral artery aneurysms presenting with acute subdural hematoma: report of two cases. No Shinkei Geka 22:577-582

Hori E, Ogiichi T, Hayashi N, Kuwayama N, Endo S (2005) Case report: acute subdural hematoma due to angiographically unvisualized ruptured aneurysm. Surg Neurol 64:144-146

Katsuno M, Murai Y, Teramoto A (2003) Acute subdural hematoma without subarachnoid hemorrhage following rupture of a distal anterior cerebral artery aneurysm: a case report. No To Shinkei 55(5):435-438

Kocak A, Ates O, Durak A, Alkan A, Cayli S, Sarac K (2009) Acute subdural hematomas caused by ruptured aneurysms: experience from a single Turkish center. Turk Neurosurg 19:333-337

Mansour O, Hassen T, Fathy S (2014) Acute aneurismal bilateral subdural haematoma without subarachnoid haemorrhage: a case report and review of the literature. Case Rep Neurol Med 2014:260853

Marbacher S, Fandino J, Lukes A (2010) Acute subdural hematoma from ruptured cerebral aneurysm. Acta Neurochir 152:501-507

Nonaka Y, Kusumoto M, Mori K, Maeda M (2000) Pure acute subdural haematoma without subarachnoid haemorrhage caused by rupture of internal carotid artery aneurysm. Acta Neurochir 142:941-944

Nozar A, Philippe D, Fabrice P, Silvia M, Marc T (2002) Acute pure spontaneous subdural haematoma from ruptured intracranial aneurysms. Interv Neuroradiol 8:393-398

Oh SY, Kwon JT, Park YS, Nam TK, Park SW, Hwang SN (2011) Clinical features of acute subdural hematomas caused by ruptured intracranial aneurysms. J Korean Neurosurg Soc 50:6-10

Ohkuma H, Shimamura N, Fujita S, Suzuki S (2003) Acute subdural hematoma caused by aneurysmal rupture: incidence and clinical features. Cerebrovasc Dis 16:171-173

O'Leary PM, Sweeny PJ (1986) Ruptured intracerebral aneurysm resulting in a subdural hematoma. Ann Emerg Med 15:944-946

O'Sullivan MG, Whyman M, Steers JW, Whittle IR, Miller JD (1994) Acute subdural haematoma secondary to ruptured intracranial aneurysm: diagnosis and management. Br J Neurosurg 8:439-445

Ragland RL et al (1993) Anterior communicating artery aneurysm rupture: an unusual cause of acute subdural hemorrhage. Surg Neurol 40(5):400-402

Schuss P, Konczalla J, Platz J, Vatter H, Seifert V, Guresir E (2013) Aneurysmrelated subarachnoid hemorrhage and acute subdural hematoma: single-center series and systematic review. J Neurosurg 118:984-990

Takada T et al (2012) Acute subdural hematoma without subarachnoid hemorrhage caused by ruptured A1-A2 junction aneurysm. Case report. Neurol Med Chir 52(6):430-434

Watanabe Ket al (1991) Ruptured distal anterior cerebral artery aneurysms presenting as acute subdural hematoma-report of three cases. Neurol Med Chir 31(8):514-517

Weil AG, McLaughlin N, Lessard-Bonaventure P, Bojanowski MW (2010) A misleading distal anterior cerebral artery aneurysm. Surg Neurol Int 1:55

Weir B, Myles T, Kahn M, Maroun F, Malloy D, Benoit B, McDermott M, Cochrane D, Mohr G, Ferguson G et al (1984) Management of acute subdural hematomas from aneurysmal rupture. Can J Neurol Sci 11:371-376

Yasui T, Komiyama M, Kishi H, Yagura H, Fu Y, Nagata Y, Tamura K (1995) Angiographic extravasation of contrast medium in acute "spontaneous" subdural hematoma. Surg Neurol 43:61-67 